\title{
Measurement of stator heat transfer in air-cooled axial flux permanent magnet machines
}

\author{
David A. Howey*, Andrew S. Holmes*, Keith R. Pullen ${ }^{\dagger}$ \\ ${ }^{*}$ Department of Electrical and Electronic Engineering, Imperial College, Exhibition Road, London, SW7 2AZ, UK \\ Tel. +44 (0)20 7594 6215, Fax. +44 (0)20 7594 6308, Email: d.howey@imperial.ac.uk \\ ${ }^{\dagger}$ School of Engineering and Mathematical Sciences, Tait Building, City University \\ Northampton Square, London, EC1V OHB, UK
}

\begin{abstract}
Relatively little fundamental research has been undertaken on heat transfer in disc type electrical machines, a common example being the axial flux permanent magnet (AFPM) machine. Power density is usually thermally limited and therefore understanding of stator convective heat transfer is crucial in the design of AFPM machines and electrical machines in general. This paper presents direct measurements of radially resolved stator convective heat transfer in a rotor-stator system, using a thin-film electrical heater array to measure heat transfer. The heater array combines surface temperature sensing with heat flux measurement. It is found for the three gap sizes considered here that average Nusselt number (non-dimensional convective heat transfer coefficient) increases from $\overline{N u} \approx 100$ at low disc speeds/rotational Reynolds numbers $\left(R e_{\theta \min }=3.7 e 4\right)$ up to $\overline{N u} \approx 300-400$ at higher speeds $\left(R e_{\theta \max }=5.6 e 5\right)$.
\end{abstract}

\section{INTRODUCTION}

Axial flux permanent magnet (AFPM) machines have been researched for a variety of applications where high efficiency, compact size and high specific power and torque are important, for example electric vehicles [1], and small wind turbine generators [2]. Electromagnetic design is well understood but less research has been undertaken on thermal aspects. The most crucial parameter governing thermal performance of AFPM machines is the convective heat transfer coefficient at the boundary between the cooling fluid and the stator surface, and the experimental measurement of these is the subject of this paper. Although the geometry discussed here is simplified in relation to a real machine, this allows comparison with previous research on rotor-stator fluid flow and provides a basic understanding of stator heat transfer.

Turbo-machinery internal air systems exhibit some fluid mechanical similarities to air-cooled disc-type electrical machines but typically it is the rotor heat transfer that is of more interest in turbo-machinery, whereas it is the stator heat transfer that is typically most important in AFPM machines.

\section{THERMAL ASPECTS OF ELECTRICAL MACHINES}

Prediction of stator temperature in an electrical machine is necessary in order to ensure sufficient cooling is available to prevent overheating at the power densities required. Losses include $I^{2} R$ (Joule) losses as well as core losses due to eddy currents and hysteresis and these result in internal heating in the stator. Steady state temperatures are governed by the rate of heat input versus the rate of heat removal. The rate of heat removal depends on the fluid mechanical design of the machine and this is a function of geometry, surface area available for heat removal and heat transfer coefficient $h$, which depends on choice of coolant fluid and fluid dynamics. Fluid temperatures also depend on coolant thermal capacity. Stator temperatures that are consistently too high will at best reduce the lifetime of the machine and at worst lead to serious failure. Prediction of operating temperature is important for a number of reasons:

1) Calculation of the machine maximum continuous rating requires an adequate thermal model since the power density is usually thermally limited in a machine of good electromagnetic design.

2) Stator temperature not only limits machine rating but also effects efficiency because the resistivity of the stator increases with temperature.

3) Copper insulating materials, polymers that may be used in stator construction and permanent magnets can only typically withstand continuous temperatures of around $150^{\circ} \mathrm{C}$ maximum.

4) Inaccurate predictions lead to the use of excessive safety margins in design. Better thermal predictions would reduce the number of prototype iterations required and help to optimise machine design and cost.

In an AFPM machine the fluid motion is driven by a pressure difference caused by the rotation of the rotor(s). Stator heat is transferred to the fluid by forced convection. The fluid velocity, pressure and temperature fields in the rotor-stator gap are not necessarily symmetrical if reflected in the $(r, \theta)$ plane and hence measured rotor heat transfer coefficients cannot be directly applied at the stator. The subject of this paper is the direct measurement of stator heat transfer coefficients. All independent and dependent variables have been non-dimensionalised so that the results can be applied to a range of AFPM machines. The local and averaged stator Nusselt numbers $N u=h R / k_{a m b}$ and $\overline{N u}=\bar{h} R / k_{a m b}$ are presented as a function of geometry, non-dimensional radial position $r / R$, rotational Reynolds number $\operatorname{Re}_{\theta}=\omega R^{2} / \nu$, gap ratio $G=g / R$. 


\section{SCALING OF AFPM MACHINES - THERMAL LIMITS}

We wish to explore how the thermally-limited output power of an AFPM machine scales with machine size. A typical AFPM machine stator includes copper coils which are electrically insulated using a thin enamel layer. The coils may also be somewhat thermally insulated from the cooling airflow by a thin layer of epoxy or plastic, if stator is mounted on plastic or potted in epoxy. A simple 1D thermal model can be constructed assuming a stator construction comprising copper with a thin layer of plastic between the copper and the cooling convective airflow. It can be shown by constructing such a model that current density $J\left(\mathrm{~A} / \mathrm{m}^{2}\right)$ within the stator is given by the following expression, where $\Delta T_{\max }$ is the maximum permissible temperature difference between stator and reference temperature (e.g. ambient), $\rho$ is the resistivity of copper, $L_{A}$ is the axial thickness of the copper in the stator, $L_{B}$ is the axial thickness of the insulating plastic, $k_{A}$ and $k_{B}$ are the thermal conductivities of copper and plastic respectively, and $h$ is the surface convective heat transfer coefficient:

$$
J=\left[\frac{\Delta T_{\max }}{\rho L_{A}\left(\frac{L_{A}}{2 k_{A}}+\frac{L_{B}}{k_{B}}+\frac{1}{h}\right)}\right]^{0.5}
$$

It is generally found that the thermal resistance of copper is substantially less than the other terms, i.e. the dominant terms are the thermal resistance of the plastic $\left(L_{B} / k_{B}\right)$, and the convective heat transfer resistance $(1 / h)$. Substituting some likely values in a real machine, say $L_{A}=1 \mathrm{~cm}, L_{B}=1$ $\mathrm{mm}, h=100 \mathrm{~W} / \mathrm{m}^{2} \mathrm{~K}, k_{A}=400 \mathrm{~W} / \mathrm{mK}, k_{B}=0.5 \mathrm{~W} / \mathrm{mK}$, it is found that $L_{A}^{2} / 2 k_{A}=1.25 \mathrm{e}-7 \mathrm{~m}^{3} \mathrm{~K} / \mathrm{W}, L_{A} L_{B} / k_{B}=2 \mathrm{e}-5$ $\mathrm{m}^{3} \mathrm{~K} / \mathrm{W}$ and $L_{A} / h=1 \mathrm{e}-4 \mathrm{~m}^{3} \mathrm{~K} / \mathrm{W}$. Therefore in this case the convection resistance dominates, although it is only five times larger than the conduction resistance due to the plastic layer. It is conceivable that in a low speed machine (with lower value of $h$ ), stator heat flow may be conduction limited rather than convection limited if there is a layer of insulating plastic or similar over the copper stator coils.

Therefore in terms of output power, in an AFPM machine which is convection limited, $J \alpha D^{-0.5}$, giving:

$$
\begin{array}{r}
P \propto J D^{4} \Omega \\
\therefore P \alpha D^{3.5} \Omega
\end{array}
$$

(In this analysis it is assumed that the stator resistance is much lower than the load resistance, i.e. $R_{s}<<R_{L}$.) The result is interesting since it shows that the power in an AFPM machine which is convection limited scales better than $D^{3}$, i.e. larger machines may be able to achieve higher specific output powers than smaller machines, for a certain value of $h$.

\section{LITERATURE REVIEW}

In recent years, much research has been undertaken on the thermal aspects of electrical machines. However, the vast majority of this has been directed at radial flux geometries such as induction motors (for example [3]). None of the results for radial flux geometries can be applied to axial flux machines since the internal fluid flow is completely different. In terms of methodologies, the lumped parameter thermal equivalent circuit method is widely used (for example [4]) in electrical machine design, in contrast to other engineering fields where CFD and FEA are routinely applied to analyse thermo-fluids problems, increasingly in an automated fashion.

Regarding the thermal aspects of axial flux geometries, Scowby et al. [5] measured pressure drop versus air mass flow rate at various speeds in an AFPM machine, and constructed a one-dimensional empirical model of fluid flow through the machine. Wang et al. [6] also developed a one-dimensional lumped parameter thermal network model using a similar empirical approach. The validity of the one-dimensional model is unclear from the experimental temperature measurements presented. In both Scowby et al. and Wang et al., the authors comment that suitable stator convective heat transfer correlations could not be found in the literature, so they apply rotor correlations (from elsewhere) to the stator.

In a similar vein, Lim et al. [7] developed a two-dimensional lumped parameter thermal model of an AFPM machine using thermal equivalent circuit networks for both the fluid flow and the stator, including thermal capacitance. Whilst lumped parameter thermal models have the advantage of being fast to solve, the approach is questionable for the modelling of the fluid domain because a gross assumption must be made about the fluid mechanics (i.e. the velocity and pressure fields in the fluid domain), and in this case it is assumed that all of the fluid flows from the centre of the machine to the outside edge and then exits. In effect the $2 \mathrm{D}$ lumped parameter approach is a simple finite element model of both the fluid and the solid. The problem is that the local spatially resolved values of the convective heat transfer coefficient $h(r)$ must be obtained elsewhere - in this case the authors use computational fluid dynamics (CFD) studies to calculate $h(r)$. There is no apparent benefit to the 2D lumped parameter approach. If it is desired to identify temperature hot spots at design stage then it would be more realistic to use a CFD code for the fluid domain, coupled directly with a numerical solution of Laplace's conduction equation in the solid domain. If however only the average operating temperature of the stator is desired, then an average value $\bar{h}$ (derived from CFD and/or experiment - the subject of this current paper) could be applied to the whole stator for a given heat input in order to find the average temperature.

Airoldi et al. [8] undertook CFD modelling of the fluid flow in an AFPM machine, with some experimental validation, using a geometric mock-up of an AFPM machine including a rotor with protrusions to simulate the effect of the permanent magnets on the airflow. The stator was a heated aluminium block. The rotor-stator system had a diameter 0.3 $\mathrm{m}$ and maximum speed $1500 \mathrm{rpm}$ (3000 rpm in CFD studies), which gives a maximum rotational Reynolds number of about $4.5 e 5$ for the CFD, and half this for the experiment. Some correlation between CFD and experiment for mass flow rates and temperatures was found, although a limited data set is presented (only one data point for mass flow rate and only four 
for temperature) so it is difficult to draw conclusions based on this. In addition, there is no comparison given between experimentally and computationally derived values of $h(r)$. In CFD simulations, a flow recirculation was observed at the periphery, but the effect of this on the lumped parameter modelling is not discussed.

There is a further aspect of this work which needs to be explored, the question of the reference temperatures used in the definition of the convective heat transfer coefficient. Airoldi et al. calculated $h$ from CFD results using the air temperature in the centre of the rotor-stator gap as the reference. However, it is recognised [9] that it is often difficult to measure the local fluid bulk temperature in rotor-stator systems. Therefore the accepted convention in these kinds of experiments is to use the ambient inlet fluid temperature as the reference temperature. Either definition is useful, but if the results from experiments are to be used predictively then it is important that a consistent definition is used. The benefit of using the fixed fluid inlet temperature is that measured values of $h$ can be used predictively to determine stator surface temperature without requiring a fluid model. However, this requires a close match of geometry and mass flow rate between the setup used for the experimental measurements and the setup used for the calculations, to which $h$ is being applied.

Marignetti et al. [10] undertook CFD modelling of an AFPM machine using a combined simulation which modelled fluid flow as well as thermal conduction in the solid domain. However, they assume without justification that the flow is laminar, and they use an experimental correlation for the stator heat transfer ('Becker') with no reference to the source of this correlation. The comparison between simulation and experiment is poor (the highest experimentally measured temperature was $130^{\circ} \mathrm{C}$ whereas the highest predicted temperature was $800^{\circ} \mathrm{C}$ at a similar operating current).

Regarding the fluid mechanics of rotor-stator disc systems, much work is available for example texts by Dorfman [11] and Owen and Rogers [9] as well as a report from ESDU [12], but little mention is made specifically of stator heat transfer. Rotorstator systems exhibit complex fluid mechanics, and there are two characteristic flow patterns. The first, Batchelor flow, is characterised by a rotating fluid core between two separate laminar or turbulent rotor and stator boundary layers. This is usually observed in enclosed systems where a recirculation of fluid from rotor to stator occurs at the periphery. In systems which are open at the periphery, or have radial throughflow, the core rotation is often diminished and instead, the fluid tangential velocity tends toward zero at the stator. This is known as Stewartson flow. In both patterns, the stator average convective heat transfer is likely to be lower than the rotor average convective heat transfer. In an AFPM electrical machine many configurations are possible: the machine may be fully enclosed, partially enclosed or open but surrounded by a grill. The rotational speed and air inlet geometry will govern the rate of air flow through the machine. It is difficult to say whether the flow will tend toward a Batchelor or Stewartson pattern since it depends on the particular machine arrangement.
In addition, some machines have magnets protruding from the rotor(s), rather than flat rotors, and these will change the flow pattern. In the experimental rig discussed here, the rotor is flat, the periphery is partially blocked and there is radial throughflow of air, which is expelled at the periphery.

\section{DESIGN OF EXPERIMENTAL APPARATUS}

\section{A. Overview}

A variety of experimental techniques are available for measuring convective heat transfer, see Rohsenow [13]. These include: thin-film heat flux gauges, infra-red or thermographic liquid crystal thermography and inverse solution of Laplace's conduction equation, thin-foil electrical heaters, and transient techniques. The method chosen here is thin-film heaters based on a printed circuit board. This has the advantage of being accurately manufactured and also, being made of copper, the resistance changes with temperature in a linear fashion allowing the heaters to be used for surface temperature measurement.

A geometric mock-up of a rotor-stator disc system corresponding to a simplified AFPM machine air-gap was constructed, shown in figure 1 . The rotor was driven at a set speed by a servo motor. The rig parameters are given in table I and figure 2 shows a photograph. The gap ratio was adjustable by insertion of machined spacers. The air exit gap at the periphery was not fully open but partially blocked, being similar in situation to most AFPM machines. Air was pumped by the rotor from an inlet at the stator centre to four exits at the edge. The air mass flow rate was measured using a bellmouth entry.

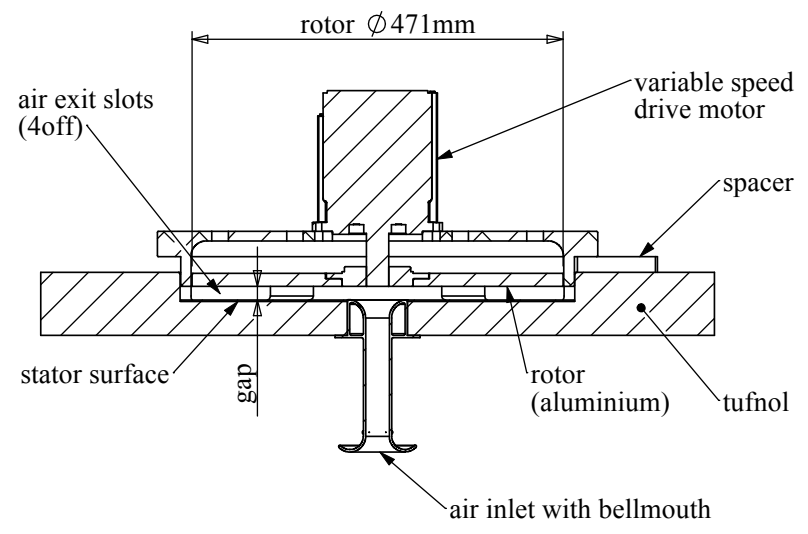

Fig. 1. Cross section through experimental apparatus

The stator heaters, heat flux measurement and temperature sensing were combined into a printed circuit board (PCB) with 14 concentric spiral shaped copper heater elements, each comprising a number of turns. The pitch of the spirals is very slight, therefore the heaters can be considered to be concentric annular rings. Two PCBs were produced: PCB1 with exposed tracks and $\mathrm{PCB} 2$ with a thin layer of epoxy (solder resist) bonded to the surface to reduce surface roughness. During experiments, an accurately known DC voltage $V$ is applied 


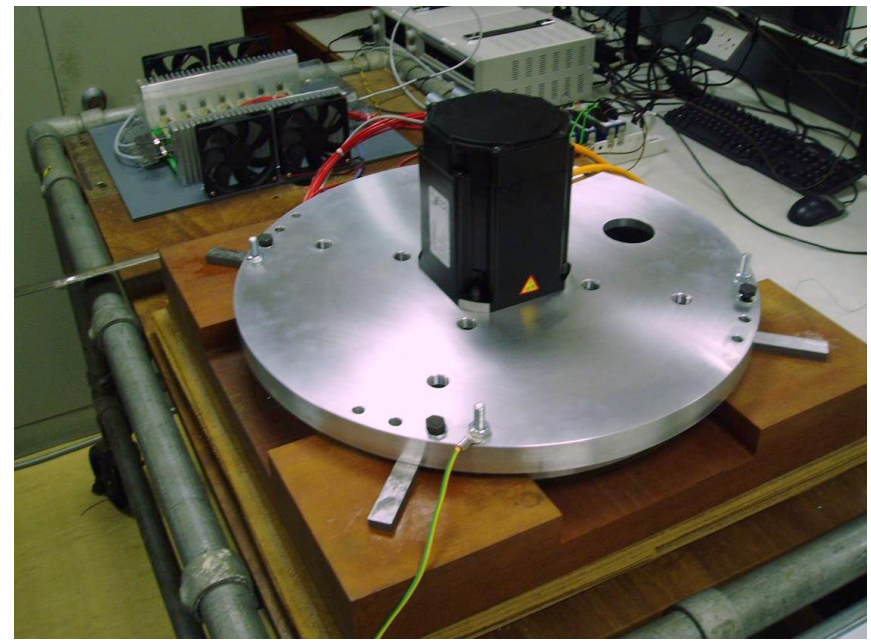

Fig. 2. Experimental rig. Two of the four air outlets can be seen, as well as the spacers and control electronics

across each heater, resulting in a current $I=V / R$ flowing which depends on the heater resistance, which depends on temperature. The total surface heat flux can be calculated using the simple formula $Q=I V$. The surface temperature can also be measured since $T=f(R)=f(V / I)$. After adjusting for radiative heat transfer, the local convective heat transfer coefficient is given as:

$$
h=\frac{Q_{n e t}}{A\left(T_{s}-T_{r e f}\right)}=\frac{q_{n e t}}{T_{s}-T_{r e f}}
$$

Strictly speaking, $T_{\text {ref }}$ should refer to the local fluid bulk temperature. However, as already discussed, the convention is to use the ambient or fluid inlet temperature as the reference temperature and this convention is followed here. Nonetheless the experimental rig also includes $0.3 \mathrm{~mm}$ diameter thermocouple probes which will be used in the future to measure fluid gap temperatures.

Closed loop control of temperature was accomplished by calibrating the heaters so that the resistance versus temperature variation was known. During measurements, a control system adjusts each heater voltage to maintain the required temperature. Each heater was designed with approximately the same resistance so that the control circuits could be identical, although the exact resistances were measured during the calibration process. By using an isothermal surface, heat transfer along the surface is negligible.

TABLE I

EXPERIMENTAL RIG PARAMETERS

\begin{tabular}{lll}
\hline \hline Rotor diameter & 471 & $\mathrm{~mm}$ \\
Maximum speed & 3000 & $\mathrm{rpm}$ \\
Stator air inlet diameter & 76.2 & $\mathrm{~mm}$ \\
Gap size $g$ & $2-22$ & $\mathrm{~mm}$ \\
Gap ratio $G$ & $0.0085-0.0934$ & \\
Number of heaters & 14 & \\
\hline
\end{tabular}

\section{B. Instrumentation and control}

The heater control system for a single channel is shown in figure 3 ; in total there are 14 identical channels.

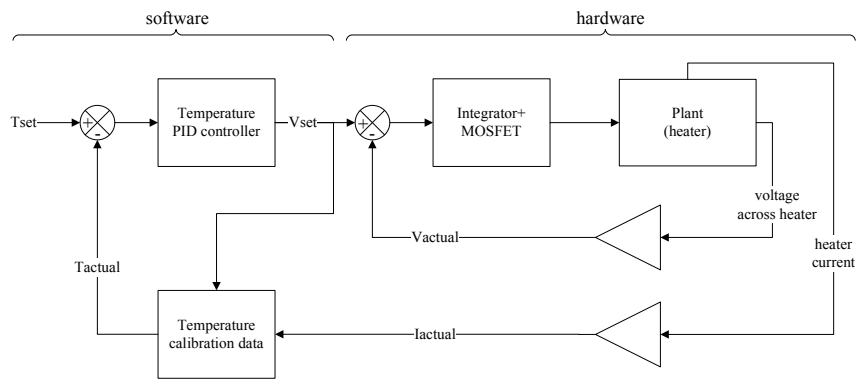

Fig. 3. Block diagram of heater control system

The voltage control feedback loop was constructed using low drift, low offset operational amplifiers. A four-wire connection to each heater enables the heater voltage to be measured accurately. This is subtracted from a reference voltage (from the DAC output). The error voltage is passed through an integrator driving a MOSFET. The temperature control feedback loop is implemented in software. Heater temperature is calculated from heater resistance using a look up table of calibration data. A PID controller responds to the temperature error, adjusting the reference voltage to control the heater to the desired temperature $T_{\text {set }}$.

\section{Calibration}

The thermocouples were calibrated against a calibrated reference thermocouple. The heater PCBs were calibrated in a temperature controlled oven and each was mounted horizontally to minimise temperature gradients. Heater resistance for each channel is given by the following equation, with $T$ measured in ${ }^{\circ} \mathrm{C}$ :

$$
R=b_{1}+b_{2} V+b_{3} T+b_{4}(V T)
$$

The resistance at $0^{\circ} \mathrm{C}$ is $b_{1} ; b_{2}$ is the change of resistance with respect to applied voltage. The change of resistance with respect to temperature is $b_{3}$, and is about $0.1 \Omega$ per ${ }^{\circ} \mathrm{C} ; b_{4}$ is a cross-correlation term which was found to be virtually negligible. From this equation, the heater temperature can be found from the applied voltage and measured current:

$$
\begin{aligned}
T & =\frac{R-\left(b_{1}+b_{2} V\right)}{b_{3}+b_{4} V} \\
\therefore T & =\frac{\frac{V}{I}-\left(b_{1}+b_{2} V\right)}{b_{3}+b_{4} V}
\end{aligned}
$$

\section{Unwanted heat losses and heat gains}

The effect of these on the results was quantified and minimised as follows: 
1) Conduction loss behind and around the circumference of the stator PCB: The heater PCB was mounted on $45 \mathrm{~mm}$ of Tufnol and $65 \mathrm{~mm}$ cork and polystyrene insulation; the maximum heat loss in this direction was calculated to be $29 \mathrm{~W} / \mathrm{m}^{2}$ which is generally small enough to be neglected.

2) Radiative heat transfer from stator to rotor: Given the relatively low stator surface temperature and low rotor-stator temperature difference, radiative heat transfer is minimal. Surface emissivities were measured with an infra-red thermal imager and found to be $0.88 \pm 0.10(95 \% \mathrm{CI})$ for PCB2 and approximately 0.4 for PCB1. The rotor emissivity was estimated at 0.05 or lower [14]. This allowed radiative heat transfer to be calculated and removed from each measurement.

3) Rotor windage heating: Rotor windage heating was estimated from experimental correlations for moment coefficient given by Owen and Haynes [15]. Based on these, drag torque and therefore windage heating on each side of the disc was calculated. For the highest speed results presented in this paper, windage heating is calculated to be $5-10 \mathrm{~W}$, about 3-5 per cent of applied heater power. The impact was minimised by allowing cooling air to circulate behind the rotor through holes in the rotor cover, and by not running high speed tests for extended periods of time.

\section{RESUlts}

Using PCB1, a set of measurements were made at rotor speeds of 100-1500 rpm and three gap sizes $g=(3.5 \mathrm{~mm}$, $5 \mathrm{~mm}, 7 \mathrm{~mm}$ ), equivalent to a range of rotational Reynolds numbers of $3.7 e 4 \leq R e_{\theta} \leq 5.6 e 5$ and gap ratios $G=$ $(0.0106,0.0212,0.0297)$. Figure 4 shows the average stator Nusselt numbers and for comparison also shows rotor average Nusselt number correlations after Dorfman [11] and Owen et al. [16] for a free rotor in the laminar and turbulent regimes. The average Nusselt numbers were not calculated across the entire stator but only for $0.6 \leq r / R \leq 1$, the area of most interest in AFPM machines. Figure 5 shows the local (radially resolved) Nusselt number measurements for $G=0.0106$ (similar figures for other gap ratios have been omitted due to space constraints but will be reported elsewhere).

\section{DISCUSSION}

As figure 4 shows, average heat transfer results are all similar for $R e_{\theta}<3 e 5$, corresponding to laminar flow where they are also very similar to Dorfman's [11] laminar flow average Nusselt numbers for an isothermal free rotor (valid for $\left.R e_{\theta}<1.8 e 5\right)$, shown in figure 4 and the following equation:

$$
\overline{N u}_{l a m}=0.35 R e_{\theta}^{0.5}
$$

On the same figure the correlation for turbulent regime average Nusselt numbers (valid for $\operatorname{Re}_{\theta}>2.8 e 5$ ) is shown for an isothermal free rotor is given by Owen, Haynes and Bayley [16]:

$$
\overline{N u}_{t u r b}=0.0151 R e_{\theta}^{0.8}
$$

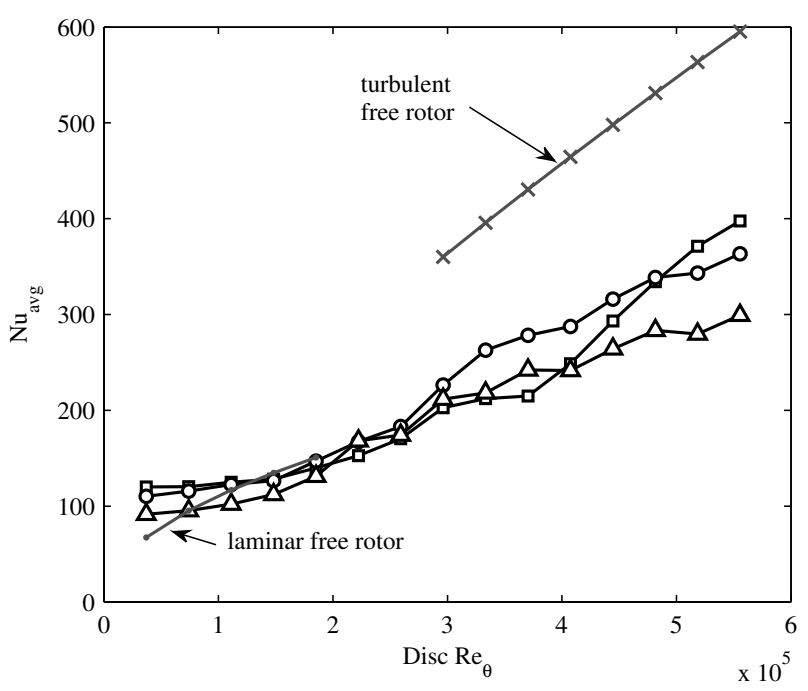

Fig. 4. $\overline{N u}$ vs. $\operatorname{Re}_{\theta}$ for three gap ratios $G=0.0106(\square), G=0.0212(\circ)$, $G=0.0297(\Delta)$, free disc $(\bullet, \times)$

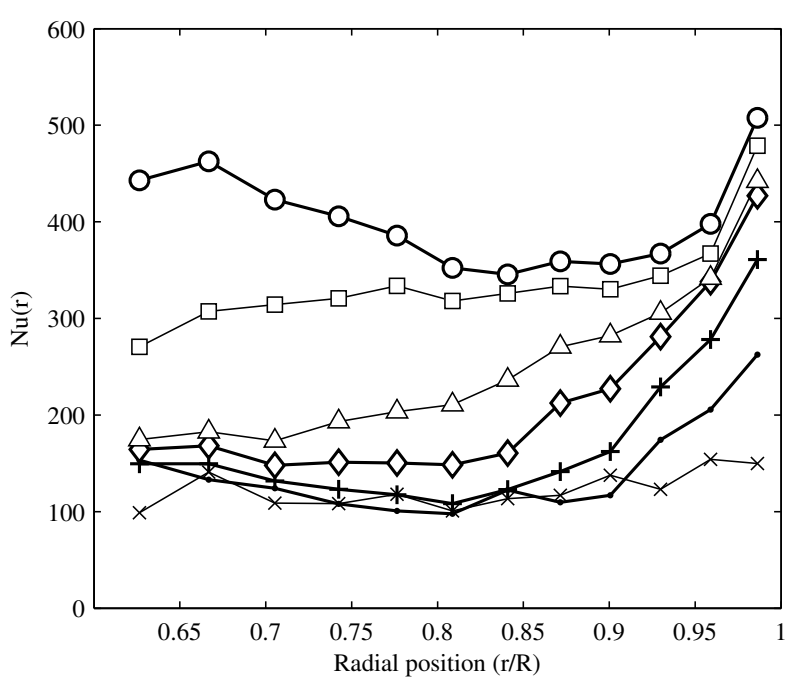

Fig. 5. $N u$ vs. $r / R, G=0.0106, R_{\theta}=3.7 \mathrm{e} 4(\times), 1.9 \mathrm{e} 5(\bullet), 2.6 \mathrm{e} 5(+)$, 3.3e5 $(\diamond), 4.1 \mathrm{e} 5(\Delta), 4.8 \mathrm{e} 5(\square), 5.6 \mathrm{e} 5(\circ) 6$

It can be seen that the measured stator heat transfer is considerably less than the free disc turbulent rotor heat transfer. This is likely caused by a lower radial and tangential velocity gradient at the stator compared to the rotor. This can be seen in the flow field imaging studies undertaken by Boutarfa and Harmand [17], where the stator radial and tangential velocities are lower than those at the rotor.

In terms of the radially resolved measurements, it can be seen in figure 5 that for $G=0.0106$, heat transfer remains steady in the laminar regime at $N u(r) \approx 100$ for $r / R<0.9$. A transition from laminar to turbulent flow begins at $\approx 3 e 5$. It is unclear where flow becomes fully turbulent and further data are required at $R e_{\theta}>5 e 5$ in order to draw conclusions.

An increase in local Nusselt numbers can be seen at 
$r / R>0.9$ for all data and it is suggested that this is caused primarily by ingress of cold (ambient temperature) air at the stator periphery. The drop in fluid bulk mean temperature increases the stator heat transfer.

\section{COMPARISON TO ACTUAL AFPM MACHINES}

Other authors [2], [18] report approximate measurements of average convective heat transfer coefficient for an entire AFPM machine during a thermal steady state test (when the machine is allowed to reach a steady state temperature under full load), these values are given in table II.

TABLE II

COMPARISON WITH MEASURED VALUES OF $h$

\begin{tabular}{llll}
\hline \hline Test conditions & $\begin{array}{l}\text { Measured } \\
\bar{h}\left(W / \mathrm{m}^{2} \mathrm{~K}\right)\end{array}$ & $\begin{array}{l}\text { Nusselt } \\
\text { number }\end{array}$ & Reference \\
\hline$\phi \approx 0.3 \mathrm{~m}$ & 150 & 975 & Spooner and \\
$\omega=3000 \mathrm{rpm}$ & & & $\begin{array}{l}\text { Chalmers 1992 } \\
{[18]}\end{array}$ \\
$R e_{\theta}=5.6 e 5$ & & 591 & Bumby and \\
$\phi \approx 0.5 \mathrm{~m}$ & 60 & & Martin 2005 \\
$\omega=500 \mathrm{rpm}$ & & & {$[2]$} \\
$R e_{\theta}=2.1 \mathrm{e} 5$ & & & \\
\hline
\end{tabular}

As can be seen the values for $\overline{N u}$ are significantly higher than those shown in figure 4, for the equivalent rotational Reynolds number. It should be noted that the method used to measure these values is not clear in either Spooner and Chalmers or Bumby and Martin so there may be considerable uncertainty. However, we suggest two key reasons for the discrepancy. Firstly, in the papers cited, the machines had rotors which were not flat and included protruding permanent magnets. These protrusions improve heat transfer by increasing fluid velocity in the gap and improving fluid mixing as well as increasing the pumped mass flow rate. Secondly, the value of $h$ may have been calculated for the entire machine based on the measured temperatures. It is conceivable that there may be significant conduction heat loss from the stator to the machine casing/support and this is lumped in to some extent with the convective heat loss from the stator surface.

In future work we aim to explore the influence of both of these factors on heat transfer predictions. In further experiments, heat transfer at higher speeds will be investigated. This should give useful information about the fluid mechanics of the air flow in the gap. In addition, conditions for a wider range of gap sizes will be measured, and mass flow rates reported.

\section{CONCLUSIONS}

Using a novel technique, measurements have been made of stator heat transfer in a partially enclosed rotor-stator disc system. Results are relevant to disc type electrical machines such as axial flux permanent magnet motors and generators. Despite the large amount of research on rotor heat transfer in rotor-stator disc systems, no previous authors have experimentally measured local stator heat transfer directly in the range of rotational Reynolds numbers and gap ratios presented here. Although the results for average Nusselt number presented here are lower than the results in real AFPM machines, this is thought to be mainly due to the lack of protrusions on the rotor. However, they still provide conservative and therefore useful estimates of stator heat transfer in air-cooled AFPM machines.

\section{ACKNOWLEDGEMENTS}

The authors would like to thank Mr Jim Ford and Mr Gary Austin at City University for their assistance with construction of the experimental rig.

\section{REFERENCES}

[1] A. Walker, Axial flux permanent magnet synchronous electric machines for hybrid electric vehicle powertrains. Imperial College, University of London, 2006.

[2] J. R. Bumby and R. Martin, "Axial-flux permanent-magnet air-cored generator for small-scale wind turbines," IEE Proceedings-Electric Power Applications, vol. 152, no. 5, pp. 1065-75, 2005.

[3] C. Micallef, S. Pickering, K. Simmons, and K. Bradley, "Improved Cooling in the End Region of a Strip-Wound Totally Enclosed FanCooled Induction Electric Machine," IEEE Transactions on Industrial Electronics, vol. 55, no. 10, pp. 3517-3524, 2008.

[4] D. Staton and A. Cavagnino, "Convection Heat Transfer and Flow Calculations Suitable for Electric Machines Thermal Models," IEEE Transactions on Industrial Electronics, vol. 55, no. 10, pp. 3509-3516, 2008.

[5] S. Scowby, R. Dobson, and M. Kamper, "Thermal modelling of an axial flux permanent magnet machine," Applied Thermal Engineering, vol. 24, no. 2, pp. 193-207, 022004.

[6] R. Wang, M. Kamper, and R. Dobson, "Development of a thermofluid model for Axial field permanent-magnet Machines," Energy Conversion, IEEE Transactions on, vol. 20, no. 1, pp. 80-87, 2005.

[7] C. Lim, J. Bumby, R. Dominy, G. Ingram, K. Mahkamov, N. Brown, A. Mebarki, and M. Shanel, "2-d lumped-parameter thermal modelling of axial flux permanent magnet generator," Proceedings of the 2008 International Conference on Electrical Machines, 2008.

[8] G. Airoldi, G. Ingram, K. Mahkamov, J. Bumby, R. Dominy, N. Brown, A. Mebarki, and M. Shanel, "Computations on heat transfer in axial flux permament magnet machines," Proceedings of the 2008 International Conference on Electrical Machines, 2008.

[9] J. Owen and R. Rogers, Flow and Heat Transfer in Rotating-Disc Systems, Vol. 1: Rotor-Stator Systems, 1st ed. Research Studies Press, 1989.

[10] F. Marignetti, V. Delli Colli, and Y. Coia, "Design of Axial Flux PM Synchronous Machines Through 3-D Coupled Electromagnetic Thermal and Fluid-Dynamical Finite-Element Analysis," IEEE Transactions on Industrial Electronics, vol. 55, no. 10, pp. 3591-3601, 2008.

[11] L. Dorfman, Hydrodynamic Resistance and the Heat Loss of Rotating Solids. Oliver \& Boyd, 1963.

[12] Engineering Sciences Data Unit, Flow in rotating components - discs, cylinders and cavities, ESDU data sheet 07004. IHS ESDU, 2007.

[13] W. Rohsenow, J. Hartnett, and Y. Cho, Handbook of Heat Transfer. McGraw-Hill Professional, 1998.

[14] A. Chapman, Fundamentals of heat transfer. Macmillan, 1987.

[15] J. Owen and C. Haynes, "Design formulae for the heat loss and frictional resistance of air cooled rotating discs," Improvements in Fluid Mechanics and Systems for Energy Conversion IV, Hoepli, Milan, pp. 127-160, 1976.

[16] J. Owen, C. Haynes, and F. Bayley, "Heat Transfer from an Air-Cooled Rotating Disk," Proceedings of the Royal Society of London. Series A, Mathematical and Physical Sciences (1934-1990), vol. 336, no. 1607, pp. 453-473, 1974.

[17] R. Boutarfa and S. Harmand, "Local convective heat transfer for laminar and turbulent flow in a rotor-stator system," Experiments in Fluids, vol. 38, no. 2, pp. 209-221, 2005.

[18] E. Spooner and B. Chalmers, "'TORUS': A slotless, toroidal-stator permanent-magnet generator," Electric Power Applications, IEE Proceedings $B$, vol. 139, no. 6, pp. 497-506, 1992. 